Journal Club

Editor's Note: These short, critical reviews of recent papers in the Journal, written exclusively by graduate students or postdoctoral fellows, are intended to summarize the important findings of the paper and provide additional insight and commentary. For more information on the format and purpose of the Journal Club, please see http://www.jneurosci.org/misc/ifa_features.shtml.

\title{
Myosin II and Dynamin Control Actin Rings to Mediate Fission during Activity-Dependent Bulk Endocytosis
}

\author{
Alexandros C. Kokotos and Darryl W. Low \\ Centre for Integrative Physiology, University of Edinburgh, EH8 9XD, Scotland, United Kingdom \\ Review of Gormal et al.
}

Neuronal communication relies on neurotransmitter-filled synaptic vesicles (SVs) fusing with the presynaptic plasma membrane during neuronal activity. After exocytosis, SVs must be retrieved locally for a continual supply of SVs and neurotransmission to be maintained. Clathrin-mediated endocytosis (CME) is the dominant mode of SV retrieval during low-intensity stimulation and is the best characterized form of endocytosis in many cell types and systems. During high-intensity stimulation, a clathrinindependent SV retrieval pathway named activity-dependent bulk endocytosis (ADBE) is also triggered (Clayton et al., 2008). The molecular mechanisms of ADBE are not well defined. In ADBE, large invaginations form from the plasma membrane and quickly undergo scission to form bulk endosomes. Subsequently, new SVs are generated from these endosomes. The large GTPase dynamin I was initially proposed to be central to ADBE triggering, because its activity-dependent dephosphorylation by calcineurin (which allows the required interaction with the membrane-bending pro-

Received March 26, 2015; revised April 24, 2015; accepted April 29, 2015.

A.C.K. is supported by the Marie-Curie Initial Training Network "Nplast" award (project number 289581) and D.W.L. by a CIVIS Prize Studentship from the University of Edinburgh. We thank Prof. M.A. Cousin for his supervision and stimulating discussion on the ADBE molecular mechanism and physiology, and Dr. J.R. Marland for his helpful discussion and critical comments on the manuscript.

The authors declare no competing financial interests.

Correspondence should be addressed to Alexandros C. Kokotos, Centre for Integrative Physiology, Hugh Robson Building, 15 George Square, University of Edinburgh, EH8 9XD, Scotland, U.K. E-mail: A.C.Kokotos@sms.ed.ac.uk.

D0I:10.1523/JNEUROSCI.1172-15.2015

Copyright $\odot 2015$ the authors $\quad 0270-6474 / 15 / 358687-02 \$ 15.00 / 0$ tein syndapin I) and its GTPase activity facilitated bulk endosome formation and scission, respectively (Clayton et al., 2009). However, these roles have recently been questioned in experiments with dynamin I, III double knock-out neurons (Wu et al., 2014). Actin networks and PI-3 kinase, which phosphorylates phosphatidylinositol 4,5-bisphosphate $\left(\mathrm{PIP}_{2}\right)$, have also been suggested to be essential for triggering ADBE (Holt et al., 2003).

Until recently, it was unknown whether ADBE was a neuron-specific pathway. In a recent issue of The Journal of Neuroscience, Gormal and colleagues (2015) demonstrated that ADBE is observed in bovine adrenal chromaffin cells, and they provided insights about the molecular mechanisms of $\mathrm{ADBE}$ triggering. In this study, the authors used the fluid phase uptake of highmolecular-weight fluorescent dextrans to specifically track ADBE. This allows specific labeling of endocytosis modes that internalize large amounts of plasma membrane, because the relatively large size of these polysaccharides makes internalization via large endosomes much more favorable compared with smaller vesicles.

Using this dextran assay, Gormal et al. (2015) demonstrated that primary chromaffin cells internalized dextran after secretion was stimulated with nicotine or $\mathrm{Ba}^{2+}$. The bulk endosomes formed had a diameter larger than $1 \mu \mathrm{m}$ and labeling with LifeAct, an actin-binding peptide, showed that the endosomes were surrounded by actin rings. Experiments using dextrans and fluorescence recovery after photobleaching (FRAP) revealed that bulk endosomes surrounded by an actin ring recovered their fluorescence over time, indicating a connection to the plasma membrane. Endosomes lacking such a ring failed to recover, indicating that fission was complete and they could no longer internalize new dextran molecules from the extracellular medium. Colocalization of LifeAct and glycosylphosphatidylinositol, a plasma membrane marker, provided further evidence that invaginating bulk endosomes were still attached to the plasma membrane. Further studies on the nature of the actin rings showed that cortical actin stress filaments partially depolymerized before ring formation and the rings formed exhibited a contractile nature. A probe that recognizes $\mathrm{PIP}_{2}$ indicated that an array of $\mathrm{PIP}_{2}$ microdomains formed before the actin rings, suggesting these microdomains may function as hotspots for actin nucleation. Although clustering of $\mathrm{PIP}_{2}$, which has an established role in $\mathrm{CME}$, was shown to precede actin ring formation, its potential mechanistic role was not further investigated.

The contractile ability of the actin rings led the authors to probe the involvement of molecular motors in providing the contraction force. Pharmacological inhibition of myosin II blocked the formation of both bulk endosomes and actin rings. The few rings which did form did not exhibit the contractile ability needed to facilitate fission. Similarly, the few bulk endosomes formed were still connected to the plasma membrane. The same phenotype was observed when dynamin was inhibited using either pharmacological or genetic appro- 
aches. In summary, this study suggests a model of an acto-myosin II ring working in tandem to constrict and initiate the fission of bulk endosomes from the plasma membrane in ADBE. These rings are induced and contracted by myosin II and dynamin.

This proposed model for the molecular mechanism of the constriction ring needs further clarification. Initial experiments indicated that dextran-positive bulk endosomes appeared before the formation of actin rings, which take part in fission. In later experiments, myosin II and dynamin inhibition prevented actin ring formation and dextran uptake. How then does ablation of the constricting actin ring result in no formation of bulk endosomes? One possibility is that dynamin and myosin II also act in early steps of endocytosis to induce the invagination of bulk endosomes and then drive the actin ring formation to facilitate fission. In support, in CME either genetic ablation or pharmacological inhibition of myosin II resulted in deficient clathrin-coated pit (CCP) invagination (Chandrasekar et al., 2014). Similarly, dynamin is recruited to nascent CCPs and overexpression of dominant-negative mutants as well as pharmacological inhibition of dynamin block CCP formation in early steps (Schmid and Frolov, 2011). In this scenario, inhibition of either protein would prevent bulk endosome formation, which would preclude actin ring assembly, as observed in this work. However, it must be noted that a direct role of actin in bulk endosome formation was not established in this study. Investigations regarding actin ring localization also revealed that it surrounds the bulk endosome, suggesting that actin participates in fission by coating the endosomes, similar to clathrin with SVs. A role in constricting the neck of invaginated membrane however, would require localization of actin specifically to this region. Combining epifluorescence and evanescent field microscopy (Merrifield et al., 2002) could reveal the precise spatiotemporal role of each molecule in bulk endosome formation and fission.

$\mathrm{ADBE}$ is a specialized form of endocytosis occurring at presynaptic terminals of neurons, and it is only activated during high-intensity stimulation. Similar to the form of endocytosis studied by Gormal et al. (2015), it retrieves large quantities of plasma membrane to compensate for the excessive amount of material added to the plasma membrane during intense SV exocytosis. However, the form of endocytosis described in chromaffin cells is distinctly different in a number of ways. Firstly, the endosomes differ in size by an order of magnitude. Secondly, the timescale of endosome fission is much slower, which allows use of FRAP in this study. Furthermore, it is unlikely that these endosomes generate new granules after their fission from the plasma membrane, since secretory granules are constantly generated from the trans-Golgi network in chromaffin cells, whereas neuronal bulk endosomes produce new functional SVs locally.

Regardless of whether the endocytosis mode described by Gormal et al. (2015) is identical to neuronal ADBE, the findings shed new light on endocytic mechanisms. With new modes of endocytosis being recently identified (Watanabe et al., 2013; Boucrot et al., 2015), it is becoming clear that some common mechanisms are conserved, especially with regards to scission of large invaginations from the plasma membrane. An example is the role of dynamin. This study escapes the recent debate as to whether the GTPase activity of dynamin is required for scission during $\mathrm{ADBE}$ and provides an alternative view on how dynamin may facilitate this process, via an additional role in the formation and function of a constricting actin ring. The discovery of a novel role for myosin II in the regulation of actin ring formation and membrane fission suggests that there are more molecules participating in endosome formation. It also creates several new questions. For example, does myosin II aid dynamin in providing motor force to constrict the bulk endosome neck, or is it directly implicated in the membrane invagination process? Inhibition of myosin II combined with fluid phase labeling with horseradish peroxidase could elucidate this at the ultrastructural level.

Further studies are required to fully understand the molecular mechanism of bulk endosome fission during ADBE. Does dynamin directly interact with actin (Gu et al., 2010) or is this achieved indirectly? In support of the first possibility, a recent study suggests that dynamin in yeast binds actin and causes reorganization of the actin network in early stages of endocytosis (Palmer et al., 2015). Alternatively, could syndapin, which links the actin cytoskeleton with membranes, be playing this role? In support of this hypothesis, both $\mathrm{PIP}_{2}$ and syndapin interact with N-WASP, which in turn activates the actin polymerization machinery Arp2/3 (Rohatgi et al., 1999; Kessels and Qualmann, 2004). Finally, are there more BAR proteins, like endophilin (Boucrot et al., 2015), which could be recruited to nascent bulk endosomes to facilitate the fission process? Much more investigation is required to fully understand the molecular mechanism of ADBE. The study by Gormal et al. (2015) highlights new avenues to explore.

\section{References}

Boucrot E, Ferreira AP, Almeida-Souza L, Debard S, Vallis Y, Howard G, Bertot L, Sauvonnet N, McMahon HT (2015) Endophilin marks and controls a clathrin-independent endocytic pathway. Nature 517:460-465. CrossRef Medline

Chandrasekar I, Goeckeler ZM, Turney SG, Wang P, Wysolmerski RB, Adelstein RS, Bridgman PC (2014) Nonmuscle myosin II is a critical regulator of clathrin-mediated endocytosis. Traffic 15:418-432. CrossRef Medline

Clayton EL, Evans GJ, Cousin MA (2008) Bulk synaptic vesicle endocytosis is rapidly triggered during strong stimulation. J Neurosci 28:6627-6632. CrossRef Medline

Clayton EL, Anggono V, Smillie KJ, Chau N, Robinson PJ, Cousin MA (2009) The phosphodependent dynamin-syndapin interaction triggers activity-dependent bulk endocytosis of synaptic vesicles. J Neurosci 29:7706-7717. CrossRef Medline

Gormal RS, Nguyen TH, Martin S, Papadopulos A, Meunier FA (2015) An acto-myosin II constricting ring initiates the fission of activitydependent bulk endosomes in neurosecretory cells. J Neurosci 35:1380-1389. CrossRef Medline

Gu C, Yaddanapudi S, Weins A, Osborn T, Reiser J, Pollak M, Hartwig J, Sever S (2010) Direct dynamin-actin interactions regulate the actin cytoskeleton. EMBO J 29:3593-3606. CrossRef Medline

Holt M, Cooke A, Wu MM, Lagnado L (2003) Bulk membrane retrieval in the synaptic terminal of retinal bipolar cells. J Neurosci 23: 1329-1339. Medline

Kessels MM, Qualmann B (2004) The syndapin protein family: linking membrane trafficking with the cytoskeleton. J Cell Sci 117:30773086. CrossRef Medline

Merrifield CJ, Feldman ME, Wan L, Almers W (2002) Imaging actin and dynamin recruitment during invagination of single clathrin-coated pits. Nat Cell Biol 4:691-698. CrossRef Medline

Palmer SE, Smaczynska-de R II, Marklew CJ, Allwood EG, Mishra R, Johnson S, Goldberg MW, Ayscough KR (2015) A dynamin-actin interaction is required for vesicle scission during endocytosis in yeast. Curr Biol 25:868878. CrossRef Medline

Rohatgi R, Ma L, Miki H, Lopez M, Kirchhausen T, Takenawa T, Kirschner MW (1999) The interaction between N-WASP and the Arp2/3 complex links Cdc42-dependent signals to actin assembly. Cell 97:221-231. CrossRef Medline

Schmid SL, Frolov VA (2011) Dynamin: functional design of a membrane fission catalyst. Annu Rev Cell Dev Biol 27:79-105. CrossRef Medline

Watanabe S, Rost BR, Camacho-Pérez M, Davis MW, Söhl-Kielczynski B, Rosenmund C, Jorgensen EM (2013) Ultrafast endocytosis at mouse hippocampal synapses. Nature 504: 242-247. CrossRef Medline

Wu Y, O'Toole ET, Girard M, Ritter B, Messa M, Liu $\mathrm{X}$, McPherson PS, Ferguson SM, De Camilli P (2014) A dynamin 1-, dynamin 3- and clathrinindependent pathway of synaptic vesicle recycling mediated by bulk endocytosis. eLife 3:e01621. CrossRef Medline 